\title{
ON SEMI-ANALYTIC AND SUBANALYTIC GEOMETRY
}

\author{
STANISŁAW ŁOJASIEWICZ \\ Instytut Matematyki, Uniwersytet Jagielloński \\ Reymonta 4, 30-059 Kraków, Poland \\ E-mail:lojasiew@im.uj.edu.pl
}

\section{Introduction}

1. The geometry discussed here takes its origin in analysing some method of solving the so called division problem.

Namely, taking into account the role which the Fourier transformation plays in the theory of linear differential operators with constant coefficients, the following question seems natural:

Let $P$ be a non-zero polynomial in $\mathbb{R}^{n}$ and let $P(D)$ denote a differential operator corresponding to it in the sense of Fourier transformation. Does there exist, for every tempered distribution $T$ (on $\mathbb{R}^{n}$ ), a tempered distribution $S$ fulfilling the equation

$$
P(D) S=T ?
$$

This question is obviously equivalent to the analogous one concerning the equation

$$
P S=T .
$$

As L. Schwartz has noticed, when we transfer, by some natural mapping, the structure of $C^{\infty}$ manifold from $n$-dimensional sphere onto the set $\Sigma=\mathbb{R}^{n} \cup \infty$, then the tempered distributions on $\mathbb{R}^{n}$ are simply exactly those ones which can be extended as distributions on $\Sigma$. Due to this elegant observation the problem proves local: it can be reduced to an analogous question about distributions on any open subset $G$ of the space $\mathbb{R}^{n}$. Moreover, we need only consider it in the case

1991 Mathematics Subject Classification: 32B20.

Lecture given at the Banach Center Colloquium on 22nd October 1992.

The paper is in final form and no version of it will be published elsewhere. 
of distributions extendible on $\mathbb{R}^{n}$, i.e. in the space $\mathcal{P}_{G}^{\prime}$ of such distributions ${ }^{1)}$.

One can get a natural generalization of the above question substituting for a polynomial $P$ any analytic function $F \not \equiv 0$ on the set $G$ (if $F$ is only of class $C^{\infty}$, the answer is evidently negative) - and in this very form was the question asked by L. Schwartz in his Theorie de distributions ([36]). The question is known under the name of division problem and has an affirmative answer, given simultaneously and independently by L. Hörmander ([14]) - for polynomials, and by the author of this paper ([16] and [17]) - for analytic functions.

Now we shall give the idea of the proof of this conjecture - the division theorem - based on a decomposition of the set

$$
Z=\{x \in Q: F(x)=0\}
$$

of zeros of function $F$ in a suitably chosen neighbourhood $Q \subset G$ of some arbitrarily fixed point $a \in G$ :

$$
Z=\bigcup_{k=0}^{n-1} \bigcup_{i} \Gamma_{i}^{k}
$$

into analytic submanifolds $\Gamma_{i}^{k}$ of dimensions $k=n-1, \ldots, 1,0$. The decomposition is required to fulfil a series of conditions, including the stratification condition: for each stratum $\Gamma_{i}^{k}$ its "boundary" should be the union of strata of dimension $<k$ of this decomposition.

A fundamental role here is played by the following inequality

$$
|F(x)| \geq c \rho(x, Z)^{N}
$$

considered in a neighbourhood of point $a$, with some constants $N>0$ and $c>0$ (here $\rho(x, Z)$ is the distance of $x$ from $Z$. The inequality holds for any analytic function and is a consequence of the more general fact, so called regular separation inequality:

$$
\rho(x, B) \geq c \rho(x, A \cap B)^{N}, \quad \text { where } x \in A
$$

for (closed) sets $A, B$ (this inequality is true locally in the case of semi-analytic $\left.\operatorname{sets}^{2}\right)$. The latter inequality is fulfilled for the closures of strata $\Gamma_{i}^{k}$ of decomposition $(*)$, and this fact also plays a fundamental role in the proof of the theorem.

Thus, let $T \in \mathcal{P}_{Q}^{\prime}$. We have to find a solution $S \in \mathcal{P}_{Q}^{\prime}$ of the equation

$$
F S=T \text {. }
$$

First, it is shown that, as a consequence of inequality (\#), we have

$$
(1 / F) T \in \mathcal{P}_{Q \backslash Z}^{\prime}
$$

This implies that it suffices to solve equation $(\square)$ for distributions $T$ whose supports are contained in $Z$. Hence, one can prove by induction that equation $(\square)$

\footnotetext{
1) This is important by technical reasons (see the idea of proof of the division theorem given later).

2) These are defined later on
} 
has a solution for distributions $T$ of support contained in the set

$$
Z^{k}=\bigcup_{j=0}^{k} \bigcup_{i} \Gamma_{i}^{j},
$$

$k=0, \ldots, n-1$. If $k=0$, then, since any distribution of support 0 has the form

$$
\sum a_{p} D^{p} \delta
$$

solving of equation $(\square)$ reduces to proving an algebraic lemma. Suppose now that we can solve equation $(\square)$ when the support of the right-hand side is contained in $Z^{k-1}$, and let $T \in \mathcal{P}_{Q}^{\prime}$ be a distribution with support in $Z^{k}$. Due to the regular separation property, it can be proved that there exists a distribution $T_{i}^{k} \in \mathcal{P}_{Q}^{\prime}$ with support in $\overline{\Gamma_{i}^{k}}$ which is equal to $T$ in a neighbourhood of a stratum $\Gamma_{i}^{k}$, and then a distribution $S_{i}^{k} \in \mathcal{P}_{Q}^{\prime}$ can be constructed, fulfilling the equation

$$
F S_{i}^{k}=T_{i}^{k}
$$

in a neighbourhood of this stratum. We conclude from the above that it suffices to solve equation $(\square)$ in the case when it has as its right-hand side a distribution

$$
T-F\left(\sum_{i} S_{i}^{k}\right)
$$

whose support is already contained in $Z^{k-1}$.

Conclusion. Analysis of construction of decomposition $(*)$ brings us to a natural supposition that strata $\Gamma_{i}^{k}$ are sets "described" by analytical inequalities. At the same time, possibility of "detachment" from a distribution $T$ of support in $Z^{k}$ its "part" of support in the set $\overline{\Gamma_{i}^{k}}$ is provided due to the regular separation property for the sets of this type which will be defined later as semi-analytic sets. Therefore we would like to have the geometry of such sets, i.e. the semi-analytic geometry, with decompositions of type $(*)$ and with the regular separation property.

2. Now we shall describe another situation which naturally involves applying the notion of semi-analytic sets.

In complex analytic geometry ${ }^{3}$ the following two beautiful theorems are true:

Theorem I. (See e.g. [25] or [26], IV.2.7.) The closure of a topological component of the set $V^{0}$ of regular points ${ }^{4)}$ of an analytic set $V$ is always an analytic set.

3) Complex (resp. real) analytic geometry - understood in the most elementary sense - deals with the properties of analytic sets, i.e. the subsets of the complex (resp. real analytic) manifold, which are locally determined by holomorphic (resp. real analytic) functions; we mean here the sets of the form $\left\{f_{1}=\cdots=f_{k}=0\right\}$, where $f_{1}, \ldots, f_{k}$ are holomorphic (resp. real analytic) functions.

4) A point of an analytic set $V$ is called a regular one, if some its neighbourhood in $V$ is a submanifold. The set of such points is denoted by $V^{0}$. 
TheOREM II. (See e.g. [25] or [26], IV.2.4.) The set $V^{*}$ of singular points ${ }^{5)}$ of an analytic set $V$ is always an analytic set.

In contrast to the complex case, in real analytic geometry none of these facts takes place. The following analytic ${ }^{6)}$ set, known under the name of Whitney's umbrella may serve as a counterexample:

$$
V=\left\{(x, y, z) \in \mathbb{R}^{3}: x^{2} z=y^{2}\right\} .
$$

The set $V^{0}$ of regular points of $V$ consists of three components having the closures:

$$
V \cap\{x \geq 0, z \geq 0\}, \quad V \cap\{x \leq 0, z \geq 0\} \quad \text { and } \quad 0 \times 0 \times(-\infty, 0] .
$$

It can be easily shown, that these closures are not analytic. Neither is analytic the set

$$
V^{*}=0 \times 0 \times[0, \infty)
$$

of singular points of $V$.

On the other hand, for any analytic set $V$ both the connected components of the set $V^{0}$ and the set $V^{*}$ are semi-analytic.

Moreover, let us notice that the set of singular points may even be a segment, e.g. for the set

$$
V=\left\{(x, y, z) \in \mathbb{R}^{3}: x^{3} z=y^{3}-3 x^{2} y\right\}
$$

we have

$$
V^{*}=0 \times 0 \times[-2,2]
$$

\section{Semi-analytic geometry}

1. Let $M$ be a real analytic manifold. We say that a subset $E \subset M$ is semianalytic if every point of $M$ has a neighbourhood $U$, such that the set $E \cap U$ is determined by a finite alternative of finite systems of analytical inequalities having the form $f>0$ or $f \geq 0$, where by $f$ we mean functions analytic in $U$.

Of course, the class of semi-analytic sets is closed with respect to the operations of complement, finite product and locally finite union. The counterimage of a semi-analytic set under the analytic map of analytic manifolds is semi-analytic ${ }^{7)}$.

When $N \subset M$ is an analytic submanifold and $E \subset N$, then semi-analyticity of the set $E$ in $N$ is not in general equivalent to its semi-analyticity in $M$, but the equivalence holds in the case when $N$ is a closed subset of the submanifold $M$.

2. To penetrate deeper the structure of semi-analytic sets, the technique of normal stratifications is applied.

\footnotetext{
5) A point of an analytic set $V$ is called a singular one, if it is not regular: a set $V^{*}$ of singular points is thus equal $V \backslash V^{0}$.

6) Even algebraic, i.e. defined by polynomials.

7) On the other hand, the operation of image (under an analytic map, under some reasonable conditions) does not in general keep semi-analyticity, but leads to a wider class of subanalytic sets (see chapter III).
} 
A normal stratification in $\mathbb{R}^{n}$ is a finite decomposition of a cube $Q=\left\{\left|x_{i}\right|<\right.$ $\left.\delta_{i}\right\}$ into semi-analytic strata, i.e. into subsets being both submanifolds and semianalytic sets; this decomposition is defined by some matrix of discriminant polynomials. This decomposition has many useful properties, most important of which is the following stratification condition: when $\Gamma$ is a stratum of dimension $k$ of this decomposition, then its "border" $(\bar{\Gamma} \backslash \Gamma) \cap Q$ is a union of some strata of dimension $<k$ of this decomposition (see [20] pp. 26-38 and [28] II.\$2).

A normal stratification at a point $a \in M$ is defined to be an image of a stratification in $\mathbb{R}^{n}$ under an inverse chart $\varphi$ such that $a=\varphi(0)$.

THEOREM ON NORMAL STRATIFICATION claims that for arbitrarily given semianalytic sets $E_{1}, \ldots, E_{k}$ and a point $a \in M$ there exists a normal at a stratification of its arbitrarily small neighbourhood $U$ which is consistent with those sets. We mean that each of the sets $E_{i} \cap U$ is a union of some strata of this stratification.

The proof of this fundamental theorem consists in constructing the matrix of discriminant polynomials, defining the desired stratification. The polynomials are obtained recursively, by means of applying to the functions describing the sets $E_{i}$ a series of operations, defined on the ground of the symmetric functions theorem.

We conclude from the above theorem that the elementary topological operations, as those of closure, interior or boundary, do preserve semi-analyticity. It should be noticed that these facts do not follow easily from the definition of semi-analyticity.

We have the following

THEOREM ON CONNECTED COMPONENTS stating that all connected components of a semi-analytic set are semi-analytic, moreover they form a locally finite family of sets.

The proof of this theorem is not quite easy. It is based on the technique of normal stratifications, where the fundamental role is played by the following

Thom Lemma. Let $P: \mathbb{R} \rightarrow \mathbb{R}$ be a polynomial of degree $\leq k$. The set

$$
\left\{t \in \mathbb{R}: \frac{d^{\nu} P}{d t^{\nu}} \in \Theta_{\nu}, \quad \nu=0, \ldots, k\right\},
$$

where each $\Theta_{\nu}$ is one of the sets

$$
(-\infty, 0), \quad\{0\} \quad \text { or } \quad(0, \infty),
$$

is an open interval, an empty set or a point.

3. A very useful tool (especially for reasoning by contradiction) appears to be the "Curve Sellecting Lemma", whose analytic version comes from Bruhat, Cartan and Wallace (see e.g. [30] p. 25, where the algebraic version is given). The proper place for this lemma seems to be in semi-analytic (and subanalytic) geometry. 
Thus, under the term of semi-analytic arc we shall mean the image of the analytic embedding of the interval $(0,1)$, which is also a relatively compact semianalytic subset of different limits in 0 and $1^{8)}$-its end-points. The closure of the arc so defined is always a simple arc of class $C^{1}$ - this is an essential property of semi-analytic arcs.

One can prove ${ }^{9)}$ the very useful

THEOREM ON THE PARAMETRIZATION OF A SEMI-ANALYTIC ARC, stating that the germ of a semi-analytic arc at its end-point is the germ of the image of the interval $(0, \varepsilon)$ under a non-constant analytic mapping of the interval $(-\varepsilon, \varepsilon)$, and conversely with a possibly smaller $\varepsilon$.

Curve Selecting Lemma. If $E \subset M$ is a semi-analytic set and $a$ is a non-isolated point of its closure $\bar{E}$, then the set $E$ contains a semi-analytic arc of the end-point a.

(See [20] pp. 103 and [28], II.6.2.)

4. We call a point of a semi-analytic set $E$ a regular point of dimension $k$ (of the set $E$ ) when some neighbourhood of this point in $E$ is an analytic submanifold of dimension $k$. It follows from the stratification theorem that the set of regular points of semi-analytic set $E$ is always dense (in $E$ ).

The dimension of the set $E$ is defined as the maximum dimension of its regular points. We shall quote here two important properties of dimension.

1) When $E \neq \emptyset$, then $\operatorname{dim}(\bar{E} \backslash E)<\operatorname{dim} E$. In particular, for the border $\partial \Gamma$ of the (non-empty) semi-analytic stratum $\Gamma$ we have the inequality $\operatorname{dim} \partial \Gamma<\operatorname{dim} \Gamma$.

2) When $f: M \rightarrow N$ is an analytic mapping of analytic manifolds and the sets $E$ and $f(E)$ are semi-analytic, then the inequality $\operatorname{dim} f(E)<\operatorname{dim} E$ holds.

THEOREM ON REGULAR POINTS asserts that the set of regular points of a semi-analytic set $E$ is semi-analytic.

The proof of this theorem is one of the most subtle proofs in semi-analytic geometry and requires applying the complexification technique (see [20] pp. 38-54 and 77-79, and [28], II. $\S 8)$.

5. A semi-analytic stratification of an analytic manifold $M$ is, by definition, a locally finite decomposition $\mathcal{T}$ of this manifold into semi-analytic strata, fulfilling the following condition: for every stratum $\Gamma \in \mathcal{T}$, its boundary $\partial \Gamma=\bar{\Gamma} \backslash \Gamma$ is a finite union of strata of family $\mathcal{T}$ having dimensions $<\operatorname{dim} \Gamma$.

Let us consider triples $(\Lambda, \Gamma, a)$, where $\Lambda, \Gamma$ are (semi-analytic) strata of a manifold $M$, such that $\Lambda \subset \partial \Gamma$ and $a \in \Lambda$. By an incidence condition we shall mean any condition imposed on such triple, which depends only on the germs of the strata $\Lambda, \Gamma$ at the point $a$.

8) Which always exist by the theorem on connected components

9) By the Puiseux Theorem (see [25] or [26], II.6.2) 
We call an incidence condition the semi-analytic one, if for every pair $\Lambda, \Gamma$ defined above, the points $a \in \Lambda$ for which the condition is fulfilled form a semianalytic set.

Well known incidence conditions are the Whitney's (a) and (b) conditions and Verdier's (w) condition. In the case of $M=\mathbb{R}^{n}$, they are formulated as follows:

$$
\delta\left(T_{a} \Lambda, T_{x} \Gamma\right) \rightarrow 0 \quad \text { when } \quad \Gamma \ni x \rightarrow a,
$$

$$
\delta\left(\mathbb{R}(x-z), T_{x} \Gamma\right) \rightarrow 0 \quad \text { when } \quad \Gamma \ni x \rightarrow a \quad \text { and } \quad \Lambda \ni z \rightarrow a,
$$

where $\delta(S, T)$ denotes the supremum of the sinus of the angle between the straight line contained in the subspace $S$ and the subspace $T$. Since all those conditions are invariant with respect to diffeomorphisms, hence they are well defined (via charts) in the case of an arbitrary analytic manifold $M^{10)}$.

It is proved that each of the above conditions is semi-analytic.

THEOREM ON SEMIANALYTIC STRATIFICATION. Let $(\gamma)$ be a semi-analytic incidence condition. For every locally finite family of semi-analytic subsets of a manifold $M$ there exists a semi-analytic stratification, consistent with this family and such, that for every pair $\Lambda, \Gamma$ of strata of this stratification, such that $\Lambda \subset \partial \Gamma$, the condition $(\gamma)$ is fulfilled at every point of the stratum $\Lambda$.

See [20], [41], [27] and [7].

TheOREm ON SEMiAnalytic tRIANGUlation. Let $\mathcal{F}$ be a locally finite family of semi-analytic subsets of a manifold $M$. Then there exists a semi-analytic stratification of $M$, which has as its strata the homeomorphic images of simplexes of a locally finite geometrical complex $K$ in the space $\mathbb{R}^{n}$ (under a homeomorphism $h:|K| \rightarrow M)^{11)}$, where all the restrictions $h_{S}: S \rightarrow h(S), S \in K$, are analytic isomorphisms.

See [19].

An incomparably more subtle notion is the Lipschitzian stratification condition formulated by T. Mostowski. By means of a theorem on existence of Lipschitzian stratifications, which he had proved earlier (for the complex case), T. Mostowski solved the old, very difficult problem of Lipschitzian equisingularization (see [31]).

The investigation of the problem in real (semi-analytic) case was continued by a student of T. Mostowski, A. Parusiński (see [32] and [33]), who proved the THEOREM ON LIPSCHITZIAN SEMI-ANALYTIC STRATIFICATIONS.

10) We have: $(\mathrm{w}) \Longrightarrow(\mathrm{b}) \Longrightarrow(\mathrm{a})$; the first implication (in which semi-analyticity is relevant!) is known as the Kuiper-Kuo theorem.

11) See later III.1. 


\section{Semi-algebraic geometry}

1. A subset of the space $\mathbb{R}^{n}$ is called semi-algebraic, when it is defined by a finite alternative of finite systems of inequalities $P>0$ or $P \geq 0$, where $P$ are polynomials on $\mathbb{R}^{n}$.

It is evident that the semi-algebraic sets form an algebra of sets. The following theorem is very important.

TARSKI-SEIDENBERG THEOREM. The image of any semi-algebraic set in $\mathbb{R}^{n} \times$ $\mathbb{R}^{n}$ under the natural projection $\mathbb{R}^{n} \times \mathbb{R}^{n}$ is semi-algebraic.

This implies that the operation of image under a polynomial mapping, and more generally - under a mapping having a semi-algebraic graph, preserves semialgebraicity.

A simple example of a consequence of this theorem is the property that the closure of a semi-algebraic set is a semi-algebraic set. Namely, it suffices to write out the definition of closure of a set and eliminate the quantifiers from it, according to the Tarski-Seidenberg theorem.

This theorem implies rather easily the following generalization. Let $M$ be an analytic manifold. A subset of the cartesian product $\mathbb{R}^{n} \times M$ is said to be partially semi-algebraic, if every point of the manifold $M$ has an open neighbourhood $U$ such that this subset is defined in $\mathbb{R}^{n} \times U$ by a finite alternative of finite systems of inequalities $F>0$ or $F \geq 0$, where $F$ are polynomials of $n$ real variables with coefficients analytic in $U$. Thus:

If the graph of a mapping of a subset of $\mathbb{R}^{n}$ into a manifold $M$ is partially semi-algebraic, then the image of every semi-analytic set (under this mapping) is semi-analytic.

R e m ark. In the triangulation theorem of II.5 the homeomorphism $h$ is partially semi-algebraic. In particular, this is the reason why the image of every simplex is semi-analytic.

Another important theorem is

TheOREM ON CONNECTED COMPONENTs. A semi-algebraic set has always a finite number of connected components and each of them is semi-algebraic.

See [20] pp. 105-112, [2] chap. 2 and [28], II.10.

2. A Nash function is defined to be a function $\varphi$, analytic in an open set of the space $\mathbb{R}^{n}$ and such that every point of this set has a neighbourhood in which $W(x, \varphi(x)) \equiv 0$ for certain polynomial $W \not \equiv 0$ on $\mathbb{R}^{n+1}$. A $k$-tuple $\left(\phi_{1}, \ldots, \phi_{k}\right)$ of Nash functions $\phi_{i}$ is called a Nash mapping.

Nash functions and mappings have a series of properties (analogous to the properties of analytic functions and mappings, among the others the implicit 
function theorem), needed to define a Nash manifold ${ }^{12)}$.

We shall mention here an interesting and useful ${ }^{13)}$ property of the Nash submanifold:

A connected Nash submanifold $\Gamma \subset \mathbb{R}^{n}$ is a semi-analytic set exactly then when its boundary $\partial \Gamma$ is a semi-analytic set.

For Nash functions we have tools (e.g. the Weierstrass preparation theorem and some techniques connected with the theorem on symmetric functions) needed for adapting in general the semi-analytic geometry, i.e. - to establish the geometry of the "semi-Nash" sets. It appears that

The "semi-Nash" subsets of an open set in $\mathbb{R}^{n}$ are exactly those ones which can be defined locally by finite alternatives of finite systems of polynomial inequalities.

Therefore those sets are called locally semi-algebraic sets.

3. The structure of Nash semi-algebraic manifold is determined ${ }^{14)}$ by the Nash semi-algebraic atlas, i.e. a Nash atlas $\left\{\varphi_{i}\right\}$, which is finite and such that the compositions are semi-algebraic, i.e. have the semi-algebraic graphs.

On the Nash semi-algebraic manifold the semi-algebraic sets can be defined as those ones whose images under all charts of the semi-algebraic Nash atlas are semi-algebraic ${ }^{15)}$

It is easy to show that on the Nash manifold the structure of Nash semialgebraic manifold is determined uniquely by the class of semi-algebraic sets.

It is proved that on a compact Nash manifold there exists the unique structure of the semi-algebraic Nash manifold ${ }^{16)}$.

Therefore on every compact Nash manifold we have a natural notion of semialgebraic set.

It refers in particular to projective spaces $P^{n}$ and more generally - to the Grassman space $\mathbf{G}_{k}^{n}$.

It appears that under the natural interpretation of $\mathbf{P}^{\mathbf{n}}$ as the projective closure of $\mathbb{R}^{n}$ (compare e.g. [24] or [25], VII.3): A subset of the space $\mathbb{R}^{n}$ is semi-algebraic exactly when it is locally semi-algebraic as a subset of the space $\mathbf{P}^{\mathbf{n}}$.

This allows us to apply the methods of semi-analytic geometry in semialgebraic geometry.

12) Remark: M. Shiota in his monograph [37] uses the term "Nash manifold" to denote the notion which will be defined in n. 3 under the name of "semi-algebraic Nash manifold".

13) It plays an important role in the proof of the semi-analytic cone theorem (see later n. 5).

14) Including the equivalence of atlases.

15) This condition is independent of the atlas (for the equivalent atlases).

16) Consistent with the structure of this Nash manifold (i.e. such one, whose atlas would be the atlas of that manifold). 
4. Let $\Gamma \subset \mathbb{R}^{n}$ be a semi-analytic stratum of dimension $k$. Consider the following tangential mapping:

$$
\tau: \Gamma \ni x \rightarrow T_{x} \Gamma \in \mathbf{G}_{k}^{n} .
$$

In the fundamentals of subanalytic geometry (see later IV.2) the essential role is played by the following

TheOrem ON TANGEntial MAPPING. A counterimage under $\tau$ of an arbitrary semi-algebraic subset of the space $\mathbf{G}_{k}^{n}$ is semi-analytic.

See [21] and [28], II prop. 10.18

5. The Chow theorem, known in complex analytic geometry, stating that any analytic subset of complex projective space must be algebraic, is proved on the grounds of Cartan-Remmert-Stein lemma, which claims that any analytic cone in $\mathbb{C}^{n}$ is algebraic (see e.g. [24] or [25], VII.6 and II.3.3). As a real analogue of this lemma we have

TheOrem ON SEMI-ANAlytic CONE. Any semi-analytic cone in $\mathbb{R}^{n}$ is semialgebraic.

(See [23] and [28], II.11.)

The above theorem implies that a projection of a semi-analytic set, even of a compact one, need not be semi-analytic. Indeed, consider the following

EXAMPLE. The set

$$
F=\left\{x=1, z=e^{y}, 0 \leq y \leq x\right\} \subset \mathbb{R}^{3}
$$

is not semi-algebraic (this follows easily from the fact that $e^{y}$ is not a Nash function). Consequently - in view of semi-algebraic cone theorem-neither the cone $[0, \infty) F$ nor the set

$$
E=[0, \infty) F \cap\{0 \leq x \leq 1\}=0 \cup\left\{z=x e^{y / x}, 0<x \leq 1,0 \leq y \leq x\right\},
$$

which is an image under the projection map $(x, y, z, u) \rightarrow(x, y, z)$ of the compact semi-analytic set

$$
\left\{y=x u, z=x e^{u}, 0 \leq x \leq 1,0 \leq u \leq 1\right\} \subset \mathbb{R}^{4},
$$

can be semi-analytic sets.

(See also [20] pp. 133-135.)

\section{Subanalytic geometry}

As we have noticed in III.5 (Example), the operation of taking image does not preserve semi-analiticity (of course, we mean here the situation where some reasonable assumptions are taken concerning the semi-analytic mapping, e.g. the assumption that its restriction to the closure of the set is a proper map). This lack of an analogue of the Tarski-Seidenberg theorem, noticed as a material inconvenience in semi-analytic geometry, leads to a rather natural idea of replacing the class of semi-analytic sets with a wider one - namely with the class of sets beeing 
locally proper projections of semi-analytic sets, in hope that it will be possible to adopt our geometry for the new class.

The first obstacle encountered on this way is connected with a quite elementary question: is the class closed with respect to the operation of completion? Though the question appears not easy to answer, the difficulty was overcome by A. M. Gabrielov in [8]. The further steps, made on the grounds of methods similar to those used in semi-analytic geometry, are rather standard.

Some years later H. Hironaka [11], who was examining the new class of sets and gave it an adequate name of "subanalytic sets", transferred on this class the results of semi-analytic geometry by means of his great desingularization theorem ${ }^{17)}$. Simultaneously R. H. Hardt in [9] introduced and investigated "analytic shadows", which later turned out to be subanalytic sets.

Since the proof of desingularization theorem is extremely difficult, it was justified to develop in subanalytic geometry indirect methods, analogous to those of semi-analytic geometry, having fulfilled thus our primary programme. Our presentation here is based upon those methods.

Let us notice that E. Bierston and P. D. Milman presented in [1] an interesting approach to this geometry, in which they obtained an indirect and not too difficult proof of uniformization and rectilinearization theorems, that form the basis for Hironaka's technique (see also H. J. Sussmann [39]).

$$
* * *
$$

1. Let $M$ be a real analytic manifold. Its subset $E$ is called subanalytic when its trace $E \cap U$ on an (open) neighbourhood $U$ of any point $x \in M$ is an image under the projection map $M \times \mathbb{R}^{k} \rightarrow M$ of a semi-analytic relatively compact subset of the manifold $M \times \mathbb{R}^{k}$ (where $k$ depends on $x$ ).

Of course, the notion of subanaliticity is of the local nature, moreover any subanalytic relatively compact set is a projection of some semi-analytic relatively compact set (the converse also is true).

It is evident that the class of subanalytic sets is closed relative to the operations of finite intersection and locally finite union of sets ${ }^{18)}$. The Cartesian product of subanalytic sets is subanalytic.

2. When considering subanalytic graphs, i.e. those of subanalytic diagrams ${ }^{19)}$, theorems on composing the mappings and on images and counterimages of subanalytic sets under such mappings require additional assumptions e.g. of relative compactness. Thus, for a subanalytic mapping $f: A \rightarrow N$, where $A \subset M$, and for a subanalytic set $E \subset M$, the image $f(E)$ is subanalytic whenever the set

\footnotetext{
17) It is recognised as one of the deepest theorems in mathematical analysis, whose existing proof - after Dieudonné - takes the second place in mathematics with regard to proof length.

18) Also relative to the operation of completion - according to the Gabrielov theorem (see later n. 4).

19) Of course, the mappings may be, in particular, analytic.
} 
$E$ is relatively compact or the mapping $f$ is "horizontally" relatively compact, i.e. $f^{-1}(W)$ is relatively compact for some neighbourhood $W$ of an arbitrary point of manifold $N$.

3. From the definition of subanalyticity there follows a series of theorems on subanalytic sets (of statements bearing a close analogy with the semi-analytic case):

TheOrem On COMPLETION ${ }^{20)}$. (See II.2.)

TheOREM On CONNECTED COMPONENTS. (See II.2.)

\section{Curve Selecting Lemma ${ }^{21)}$.}

In the analogous TANGential MAPPING TheOREM (see III.4), graph of the mapping is simply subanalytic. The proof is easier; it suffices to write out suitably the definition of the graph ${ }^{22)}$.

4. In the sequel, some lemmas of technical character are involved which enable representing subanalytic compact sets as finite unions of semi-analytic strata projections, such that the projection maps have constant rank or even are immersions - moreover, some restrictions are fulfilled relative to tangents variations for those strata.

Having got a series of properties of dimension, analogous to the semi-analytic case (see II.4), we can prove the

GABRIElov TheOREM ON COMPLEMENT. The complement of any subanalytic set is subanalytic.

Very useful, from the technical point of view, is the

STASICA LEMma [38], according to which any relatively compact subanalytic stratum

$$
\Gamma \subset M \times N
$$

such that the restriction $\pi_{\Gamma}: \Gamma \rightarrow M$ of natural projection map $\pi: M \times N \rightarrow M$ is an immersion, admits the decomposition

$$
\Gamma=\Gamma_{1} \cup \cdots \cup \Gamma_{m} \cup F,
$$

in which $F$ is a subanalytic set of dimension $<\operatorname{dim} \Gamma$, and every $\Gamma_{i}$ is an open subanalytic $($ in $M \times N)$ subset of the stratum $\Gamma$, whose projection $\Delta_{i}=\pi\left(\Gamma_{i}\right)$ is a subanalytic stratum, where $\Gamma_{i}$ is the graph of the analytic mapping $\Delta_{i} \rightarrow N$.

5. Analogously, there holds the Theorem on Regular Points (comp. II.4) which is not easy to prove. This theorem has been proved by M. Tamm [40], who

20) And on other elementary topological operations.

21) It deals with semi-analytic arcs: "subanalytic arc" must also be semi-analytic (see later n. 8).

22) The same holds in the semi-analytic case. 
used Hironaka's desingularization theorem, and by K. Kurdyka [15], who found a direct proof.

6. Undoubtly, the most difficult of the facts proved by now in subanalytic geometry (some tens pages of proof!) is very natural PAWŁUCKI's THEOREM (see. [35]), of simple and elementary statement. Namely, for a subanalytic set $E \subset M$, let us call a point $x \in M$ essentially subanalytic, if the trace $E \cap U$ is not semi-analytic for any neighbourhood of this point. Thus,

PAWŁUCKI'S THEOREM states that for an arbitrary subanalytic set $E$, the set of its essentially subanalytic points is subanalytic.

7. We have the important

Gabrielov Theorem on COMPonents of A Fibre. Namely: if $E \subset M \times N$ is a relatively compact subanalytic set, then the number of components of the fibre $E_{x}=\{y:(x, y) \in E\}$ remains bounded for $x \in M$.

The proof of this theorem is not easy. It suffices however to prove it for the semi-analytic case, which can be done by induction with respect to

$$
k=\max \operatorname{dim} E_{x}
$$

on the grounds of normal stratification properties (see II.2).

8. TheOREM ON SUbANALYTIC SETS OF SMALL DimENSiOn states that any subanalytic set of dimension $\leq 1$, as well as any subanalytic subset of a manifold of dimension $\leq 2$, is necessarily semi-analytic.

This theorem rather easily follows from the properties of semi-analytic arcs (see II.2) and from the conclusion of Tarski-Seidenberg theorem on projecting semi-algebraic sets (see III.1).

9. Basing on the theorem from n. 8 one can relatively easily prove theorems on the so called Regular Separation:

I. Let $A$ and $B$ be subanalytic compact subsets of the space $\mathbb{R}^{n}$. Then

$$
\rho(x, B) \geq d \rho(x, A \cap B)^{N} \quad \text { for } x \in A
$$

with some constant $d>0$ and some exponent $N>0$.

II. Let $f: A \rightarrow \mathbb{R}$ be a subanalytic function continuous on a compact set $A \subset \mathbb{R}^{n}$. Then

$$
|f(x)| \geq d \rho(x, Z)^{N} \quad \text { for } x \in A
$$

with some constant $d>0$ and some exponent $N>0$, where

$$
Z=\{x: f(x)=0\} .
$$

The latter theorem is in fact a particular case of the former. 
One can relatively easily notice that the second theorem for analytic functions is equivalent to the following

INEQUALITY.

$$
|\operatorname{grad} g(x)| \geq|g(x)|^{\Theta} \quad \text { in the neighbourhood of zero, }
$$

for any analytic function $g \not \equiv 0$ in the neighbourhood of 0 in $\mathbb{R}^{n}$, with some exponent $\Theta$ such that

$$
0<\Theta<1
$$

This inequality is used in the proof of the

THEOREM ON LIMITS OF TRAJECTORIES of a dynamical system

$$
\dot{x}=-\operatorname{grad} f(x),
$$

where $f \geq 0$ is analytic in a neighbourhood of zero of the $\mathbb{R}^{n}$ space. Namely, each of the trajectories $x(t)$, starting from the points sufficiently near to zero, has a limit when $t \rightarrow \infty$.

The following TANGENT PROBLEM, formulated by Thom more than twenty years ago, remains unsolved until nowadays:

Do the trajectories also have tangent limits?

10. We will complete this paper with the remark that there remain true, both in subanalytic an in semi-algebraic case, the THEOREMS ON STRATIFICATION AND TRIANGULATION (see II.5).

Proofs of the stratification theorems with incidence conditions (e.g. with Whitney's and Verdier's conditions; see II.5) are valid unchanged. Much more subtle THEOREM ON LIPSCHITZIAN SUBANALYTIC STRATIFICATION was recently proved by A. Parusiński (see [34]).

The proof of the triangulation theorem in the subanalytic and semi-algebraic cases is much easier (see [13] and [24]), because when reasoning by induction relative to space dimension, we do not need to choose the directions of projecting, unlike in the semi-analytic case where we face with lack of the Tarski-Seidenberg theorem (see [19]).

\section{References}

[1] E. Bierstone, P. D. Milman, Semi-analytic and Subanalytic Sets, Publications de l'IHES, 67 (1988), pp. 5-42.

[2] J. Bochnak, M. Coste, M.-F. Roy, Géométrie algébrique réelle, Springer 1987.

[3] M. Coste, M.-F. Roy, Encore une démonstration de l'existence des stratifications satisfaisantes la condition (w) de Verdier, Bull. Acad. Sci. Pol. 37 (1989), pp. 597-601.

[4] Z. Denkowska, S. Łojasiewicz, J. Stasica, Certaines propriétés élémentaires des ensembles sous-analytiques, Bull. Acad. Sci. Pol. 27 (1979), pp. 529-536. 
[5] - Sur le théorème du complementaire pour les ensembles sous-analytiques, ibid., pp. 537-539.

[6] - Sur le nombre des composantes connexes de la section d'un sous-analytique, ibid. 30 (1982), pp. 333-335.

[7] Z. Denkowska, K. Wachta, Une construction de la stratification avec la condition (w), ibid. 35 (1987), pp. 401-405.

[8] A. M. Gabrielov, Projections of semianalytic sets, Funkc. Analiz i ego Priloz. $2 \mathrm{n}^{\mathrm{O}} 4$ (1969), pp. 18-30.

[9] R. Hardt, Stratification of real analytic maps and images, preprint (about 1973).

[10] - Triangulation of subanalytic sets and proper light subanalytic maps, Invent. Math. 38 (1977), pp. 207-217.

[11] H. Hironaka, Subanalytic sets, Number Theory in Honor of Y. Akizuki, Kinokuniya, Tokyo (1973).

[12] , Introduction to real analytic sets and real analytic maps, Univ. di Pisa (1973).

[13] , Triangulation of algebraic sets, Proceedings of Symp. in Pure Math., vol. 29 (1975).

14] L. Hörmander, On the division of distributions by polynomials, Arkiv för Matematik 3 (1958), pp. 555-568.

[15] K. Kurdyka, Points réguliers d'un ensemble sous-analytique, Ann. Inst. Fourier (Grenoble) 38 (1988), pp. 133-156.

[16] S. Łojasiewicz, Division d'une distribution par une fonction analytique des variables réelles, C. R. Paris 246 (1958) pp. 683-686.

[17] _ Sur le problème de la division, Studia Math. 18 (1959), pp. 87-136; and Rozprawy Matem. 22 (1961).

[18] Une propriété topologique des ensembles analytiques réels, Coll. du C.N.R.S. sur les équations aux derivées partielles, Paris 1962, pp. 87-89.

[19] - Triangulation of semi-analytic sets, Ann. Scuola Norm. Sup. di Pisa, ser. 3 18.4 (1964), pp. 449-474.

[20] — Ensembles semi-analytiques, IHES 1965.

[21] _ Sur la semi-analycité des images inverse par l'application-tangente, Bull. Acad. Sci. Pol. 17 (1979), pp. 525-527.

[22] — Sur la séparation régulière, Seminari Geometria, Bologna 1985, pp. 119-121.

[23] _ Sur les cônes semi-analytiques, ibid., pp. 123-125.

[24] - Stratifications et triangulations sous-analytiques, ibid. 1986, pp. 83-97.

[25] — Wstȩp do geometrii analitycznej zespolonej, PWN, Warszawa 1988.

[26] - Introduction to Complex Analytic Geometry, Birkhaüser, Basel 1991.

[27] S. Łojasiewicz, J. Stasica, K. Wachta, Stratifications sous-analytiques. Condition de Verdier, Bull. Acad. Sci. Pol. 36 (1986), pp. 531-539; and Seminari di Geometria, Bologna 1988-1991,pp. 83-88.

[28] S. Łojasiewicz, M. A. Zurro, Una introducción a la geometria semi- y sub-analitica, Univ. de Valladolid 1993.

[29] B. Malgrange, Ideals of differentiable functions, Oxford: Univ. Press 1966.

[30] J. Milnor, Singular points of complex hypersurfaces, Annals of Math. Studies 61, 1968.

[31] T. Mostowski, Lipschitz equisingularity, Dissertationes Mathematicae (Rozprawy Matem.) 243 (1985)

[32] A. Parusiński, Lipschitz stratification of real analytic sets, Singularities, Banach Center Publ. XX, Warsaw (1988), pp. 323-333.

[33] - Lipschitz properties of semianalytic sets, Ann. Inst. Fourier (Grenoble) 38,4 (1988), pp. 189-213. 
[34] , Lipschitz stratification of subanalytic sets, Ann. Ecole Norm. Sup. (to appear).

[35] W. Pawłucki, Points de Nash des ensembles sous-analytiques, Memoirs Am. Math. Soc. 425 (1990).

[36] L. Schwartz, Theorie des distributions, Paris 1957-1958.

[37] M. Shiota, Nash Manifolds, Springer Lecture Notes Math. 1269 (1987).

[38] J. Stasica, The Whitney condition for subanalytic sets, Zesz. Nauk. U.J., Prace Mat. 32 (1982), pp. 211-221.

[39] H. J. Sussman, Real-analytic desingularisation and subanalytic sets: an elementary approach, Trans. Amer. Nath. Soc. 317 (1990), pp. 417-461.

[40] M. Tamm, Subanalytic sets in calculus of variations, Acta Math.Uppsala 146(1981), pp. 167-199.

[41] J.-L. Verdier, Stratifications de Whitney et le théorème de Bertini-Sard, Inventiones Math. 36 (1976), pp. 259-312. 